\title{
Optical characterization of pseudomorphic AlGaAs/InGaAs/GaAs heterostructures
}

\author{
Z.Ya. Zhuchenko, G.G. Tarasov, S.R. Lavorik, Yu.I. Mazur, M.Ya. Valakh, H. Kissel*, \\ W.T. Masselink*, U. Mueller*, C. Walther* \\ Institute of Semiconductor Physics, NAS of Ukraine, 45, Prospect Nauki, 252650 Kiev, Ukraine \\ *Department of Physics, Humboldt-Universitat zu Berlin, Invalidenstrasse 110, D-10115 Berlin, Germany
}

\begin{abstract}
Pseudomorphic strained-layer $\mathrm{Al}_{\mathrm{x}} \mathrm{Ga}_{1-\mathrm{x}} \mathrm{As} / \mathrm{In}_{\mathrm{y}} \mathrm{Ga}_{1-\mathrm{y}} \mathrm{As} / \mathrm{GaAs}$ heterostructures have been studied by means of photoluminescence and Raman scattering. It is established the correlation between the photoluminescence line shape changes and the Raman spectra modification when the quantum well width is below the critical layer thickness estimated to be of $25 \mathrm{~nm}$ for $y=0.1$. The photoluminescence feature observed for the InGaAs quantum well width equal to $20 \mathrm{~nm}$ as extremely narrow exciton-like peak with the full width at half of maximum equal to $1.5 \mathrm{meV}$ at low temperature $(T=6 \mathrm{~K})$ transforms into broad band of the full width at half of maximum equal to $16 \mathrm{meV}$ when the quantum well width reaches the value about of $12 \mathrm{~nm}$. The photoluminescence line shape broadening is accompanied by the modifications of Raman spectra. A new line arising at the spectral position $v=160 \mathrm{~cm}^{-1}$ is assigned to impurity-induced longitudinal acoustic mode of $\mathrm{In}_{\mathrm{y}} \mathrm{Ga}_{1-\mathrm{y}}$ As. The changes observed in optical spectra are related to the generation of defects in the under-critical layer thickness region.
\end{abstract}

Keywords: heterostructures, critical layer thickness, photoluminescence, Raman scattering, quantum well.

Paper received 08.10.99; revised manuscript received 13.10.99; accepted for publication 18.10.99.

\section{Introduction}

Pseudomorphic modulation-doped $\mathrm{Al}_{1-\mathrm{x}} \mathrm{Ga}_{\mathrm{x}} \mathrm{As} / \mathrm{In}_{\mathrm{y}} \mathrm{Ga}_{1-\mathrm{y}} \mathrm{As} /$ $\mathrm{GaAs}$ heterostructures contain the strained semiconductor layer due to the 6-7\% mismatch between the lattice constants in GaAs and InAs [1]. The strain leads to accumulation of elastic energy which can be partly released through the generation of various defects [2]. The relaxation threshold depends on the growth conditions (temperature, growth rate, substrate orientation) [3], In content and is characterized by certain critical layer thickness (CLT) [4], which in turn is equivalent to a critical strain energy. A substantial amount of work has been done in characterizing InGaAs/GaAs single quantum wells (QW) [5-7] and the results were always related to CLT. When the layer thickness is significantly larger than the critical one the formation of misfit dislocations generated at the interface or at the surface is expected which are believed to penetrate the entire layer thickness, releasing the accumulated strain energy. This simplified picture was argued by Brafman et al. [8], who suggested that the dislocations do not diffuse through the entire layer, and the defects are not mobile. The step-like profile of the strain was assumed: a lower strained thin layer is formed and the growth continues preserving the larger lattice parameter until the elastic energy becomes too large and the next step takes place.
Below the CLT a high quality homogeneously strained layers free of misfit dislocations are expected. A strong single and narrow excitonic emission line from the QW is the typical feature of the photoluminescence (PL) spectrum in this region of QW thicknesses which reflects a high interfacial quality also [9]. The reported values of CLT are markedly scattered being determined by different experimental techniques (PL, X-ray (double crystal) diffraction, Hall effect, transmission electron microscopy, reflection high energy electron diffraction, cathodoluminescence or Raman scattering) due to either the different resolution of method or different criteria used to derive the CLT. For InGaAs heterostructures the CLT dependence is strongly determined by the mole indium fraction and reveals a remarkable change at $y \sim 0.35$. In particular case of $y=0.1$ the CLT was estimated to be of $25 \mathrm{~nm}$ [10]. Indeed the PL measurements for the QW of $20 \mathrm{~nm}$ width have shown an exciton-like peak accompanied by a weak and comparatively broad low-energy structure, ascribed to the two-dimensional-electron gas (2DEG)-hole bound to acceptor transitions. However further diminishing the InGaAs layer thickness is expected to result in a strong broadening of the PL feature in the under-CLT region. This broadening can not be ascribed to the dislocation generation and has to be connected with potential fluctuations, indium content varia- 
tion and interfacial roughness. By means of nondestructive (PL and Raman scattering) study we intend to trace here the correlation between the PL line shape and behavior of phonon spectra of pseudomorphic modulation-doped $\mathrm{Al}_{1-\mathrm{x}} \mathrm{Ga}_{\mathrm{x}} \mathrm{As} / \mathrm{In}_{\mathrm{y}} \mathrm{Ga}_{1-\mathrm{y}} \mathrm{As} / \mathrm{GaAs}$ heterostructures.

\section{Experimental details}

The samples used in this investigation are pseudomorphic $\mathrm{In}_{\mathrm{y}} \mathrm{Ga}_{1-\mathrm{y}} \mathrm{As}$ modulation-doped quantum wells grown on semi-insulating, (100)-oriented GaAs substrates in a Riber 32-P gas-source MBE (GSMBE) system. The typical epitaxial layer sequence consists of a GaAs buffer layer, an undoped $\mathrm{In}_{\mathrm{y}} \mathrm{Ga}_{1-\mathrm{y}}$ As strained QW (2DEG channel), an $\mathrm{Al}_{\mathrm{x}} \mathrm{Ga}_{1-\mathrm{x}} \mathrm{As}$ undoped spacer, an $\mathrm{Al}_{\mathrm{x}} \mathrm{Ga}_{1-\mathrm{x}} \mathrm{As}$ heavily $\mathrm{Si}$ doped supplier layer, and a $\mathrm{Si}$-doped $\mathrm{GaAs}$ cap layer. The range of $\mathrm{Al}_{\mathrm{x}} \mathrm{Ga}_{1-\mathrm{x}} \mathrm{As}$ and $\mathrm{In}_{\mathrm{y}} \mathrm{Ga}_{1-\mathrm{y}} \mathrm{As}$ layer compositions spreads into $0.15 \leq x \leq 0.20$ and $0.08 \leq y \leq 0.20$, respectively. Double-crystal X-ray diffraction and simulation of the double X-ray rocking curve were used to verify the samples' structural parameters. The measurements were carried out with a Bede Q1B X-ray diffractometer using the symmetric (004) reflex. The X-ray patterns were indicative of the high quality of the structures under investigation. The structural parameters of the samples determined from this study are listed in Table 1 together with the electron densities $N_{\mathrm{s}}$ and the dc mobility $\mu_{\mathrm{dc}}$ of the $2 \mathrm{D}$ electrons, obtained from the low-field Hall measurements down to $4.2 \mathrm{~K}$. The PL was excited by the $514.5 \mathrm{~nm}$ line of a cw $\mathrm{Ar}^{+}$laser. The PL signal was dispersed through a 3/4-m Czerny-Turner scanning spectrometer, with a spectral resolution better than $0.1 \mathrm{meV}$, and detected using phase-sensitive detection with a thermoelectrically cooled photomultiplier tube (containing a $\mathrm{GaAs}(\mathrm{Cs})$ photocathode) or a $\mathrm{LN}_{2}$ cooled high-purity $\mathrm{Ge}$ detector. The samples were mounted in an Oxford Spectromag 4000 system, which allows measurements in magnetic fields up to $7 \mathrm{~T}$ and at temperatures from 1.7 to $300 \mathrm{~K}$.

Raman scattering studies were performed using the 514.5 $\mathrm{nm}$ line of argon-ion laser. The room-temperature measurements in backscattering geometry were carried out

Table 1. Sample Parameters.

\begin{tabular}{|c|c|c|c|c|}
\hline Samples & & S1 & S2 & S3 \\
\hline $\begin{array}{c}\text { GaAs:Si } \\
\text { (cap layer) }\end{array}$ & $\begin{array}{c}d(\mathrm{~nm}) \\
N_{D}\left(10^{18} \mathrm{~cm}^{-3}\right)\end{array}$ & $\begin{array}{c}5 \\
2.5\end{array}$ & $\begin{array}{c}5 \\
2.5 \\
\end{array}$ & $\begin{array}{c}5 \\
2.5\end{array}$ \\
\hline $\mathrm{Al}_{\mathrm{x}} \mathrm{Ga}_{1-\mathrm{x}} \mathrm{As}: \mathrm{Si}$ & $d(\mathrm{~nm})$ & 35 & 35 & 35 \\
\hline (supplier layer) & $\begin{array}{c}x \\
N_{D}\left(10^{18} \mathrm{~cm}^{-3}\right)\end{array}$ & $\begin{array}{c}0.20 \\
2.0\end{array}$ & $\begin{array}{c}0.20 \\
0.2\end{array}$ & $\begin{array}{c}0.20 \\
0.2\end{array}$ \\
\hline $\begin{array}{c}\mathrm{Al}_{\mathrm{x}} \mathrm{Ga}_{1-\mathrm{x}} \mathrm{As} \\
\text { (spacer layer) }\end{array}$ & $\begin{array}{c}d(\mathrm{~nm}) \\
x\end{array}$ & $\begin{array}{c}7.5 \\
0.20\end{array}$ & $\begin{array}{c}7.5 \\
0.20\end{array}$ & $\begin{array}{c}7.5 \\
0.20\end{array}$ \\
\hline $\begin{array}{c}\operatorname{In}_{y} \mathrm{Ga}_{1-y} \mathrm{Ass} \\
\text { (quantum well) }\end{array}$ & $\begin{array}{c}d(\mathrm{~nm}) \\
y\end{array}$ & $\begin{array}{c}20 \\
0.10\end{array}$ & $\begin{array}{c}15 \\
0.10\end{array}$ & $\begin{array}{c}12 \\
0.10\end{array}$ \\
\hline $\begin{array}{c}\text { GaAs } \\
\text { (buffer layer) }\end{array}$ & $d(\mathrm{~nm})$ & 200 & 200 & 200 \\
\hline $\begin{array}{l}\text { Hall data } \\
(\text { at } 12 \mathrm{~K}) \text { : }\end{array}$ & $\begin{array}{c}N_{S}\left(10^{11} \mathrm{~cm}^{-2}\right) \\
\mu_{\mathrm{dc}}\left(10^{3} \mathrm{~cm}^{2} \mathrm{~V}^{-1} \mathrm{~s}^{-1}\right)\end{array}$ & $\begin{array}{c}7.02 \\
59\end{array}$ & $\begin{array}{c}2.5 \\
3\end{array}$ & $\begin{array}{l}6.3 \\
40\end{array}$ \\
\hline
\end{tabular}

within the spectral range $100-400 \mathrm{~cm}^{-1}$. The scattered light was analyzed with a double monochromator and detected by photon-counting electronics. In order to avoid the sample overheating the laser radiation was focused through a cylindrical lens.

\section{Experimental results and discussion}

\section{A. PL study}

The PL spectra for $\mathrm{Al}_{\mathrm{x}} \mathrm{Ga}_{1-\mathrm{x}} \mathrm{As} / \mathrm{In}_{\mathrm{y}} \mathrm{Ga}_{1-\mathrm{y}} \mathrm{As} / \mathrm{GaAs}$ heterostructures with varying $\mathrm{QW}$ width were measured at low temperature $T=6 \mathrm{~K}$ and at the same excitation intensity. Typically the spectral line shape changes with the intensity increase due to hot carrier contribution, however no noticeable shift of the PL peak is observed in the samples under consideration. Fig. 1 shows the single-peaked PL spectra for three representative samples with the InGaAs QW width equal to $20 \mathrm{~nm}(\mathrm{~S} 1), 15 \mathrm{~nm}$ (S2), and $12 \mathrm{~nm}$ (S3), respectively. The Si-doping in $\mathrm{Al}_{\mathrm{x}} \mathrm{Ga}_{1-\mathrm{x}}$ As layer provides the corresponding $\mathrm{QW}$ conduction band filling with electrons of

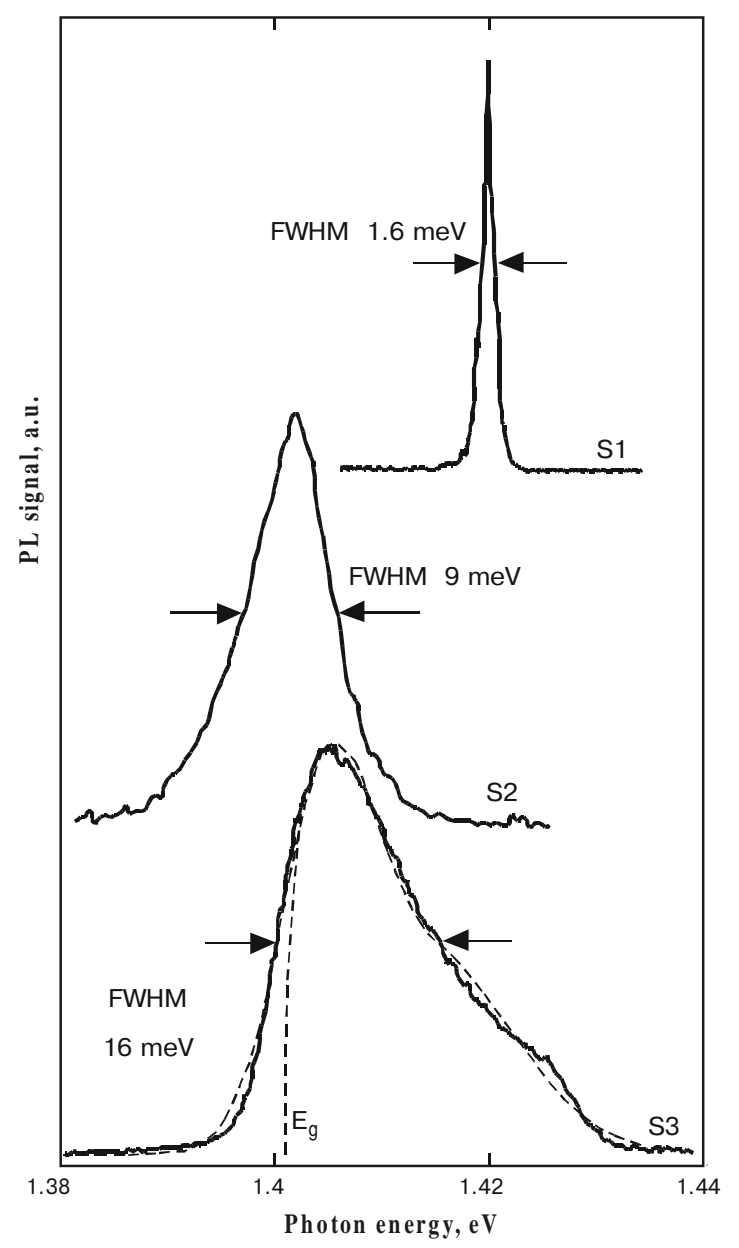

Fig. 1. Low-temperature PL from three representative samples of pseudomorphic modulation-doped $\mathrm{Al}_{1-\mathrm{x}} \mathrm{Ga}_{\mathrm{x}} \mathrm{As} / \mathrm{In}_{\mathrm{y}} \mathrm{Ga}_{1-\mathrm{y}} \mathrm{As} / \mathrm{GaAs}$ heterostructures with different InGaAs QW widths at $T=6 \mathrm{~K}$. The fit to Eq. (1) is shown by dashed line. 


\section{Z.Ya. Zhuchenko et al.: Optical characterization of pseudomorphic heterostructures...}

the sheet density $N_{s}=7.02 \cdot 10^{11} \mathrm{~cm}^{-2}, N_{s}=2.50 \cdot 10^{11} \mathrm{~cm}^{-2}$, and $N_{s}=6.30 \cdot 10^{11} \mathrm{~cm}^{-2}$ found from the Hall measurements (see Table 1). The presence of the 2DEG modifies strongly the potential profile at $\mathrm{AlGaAs} / \mathrm{InGaAs}$ interface and gives rise the energy levels falling down deeper in comparison with the case of empty QW. Due to strain the valence band is split, the light hole subband being the order of $60 \mathrm{meV}$ below the heavy hole subband. Nominally the direct transitions between the $n=1$ electron subband and the $n=1$ heavy hole subband with the momentum conservation are allowed. In fact the photoexcited holes rapidly relax to the top of valence band, and the transitions of the carriers with $k \sim 0$ are actual only.

This case is represented by Fig. 1, where very narrow, with the full width at half maximum (FWHM) $\sim 1.6 \mathrm{meV}$, PL peak is observed (Sample S1). This exciton-like feature proves the high quality both of the QW and of the interfaces. Indeed, the presence of the 2DEG of sufficiently high density $\left(N_{s}=7.02 \cdot 10^{11} \mathrm{~cm}^{-2}\right)$ would result in broadening the corresponding PL feature due to breaking the momentum conservation law, caused by the available defects and imperfections. Typically this breakage leads to the all electrons with $k$ values spread up to $k=k_{F} \sim 2 \cdot 10^{6} \mathrm{~cm}^{-1}$ become involved into the optical transitions and the PL feature is tailed up to the Fermi energy $\left(E_{F}\right)$. Using the simple relation between the $\left(E_{F}-E_{1 e}\right)=\pi \hbar^{2} N_{s} / m_{e}{ }^{*}$ for the case with the single occupied electron subband $\left(E_{1 e}\right)$ one can find $E_{F}=23 \mathrm{meV}$ if the electron effective mass $\left(m_{e}{ }^{*}\right)$ in a strained-layer $\mathrm{In}_{\mathrm{y}} \mathrm{Ga}_{1-\mathrm{y}} \mathrm{As} \mathrm{QW}$ with $y \approx 0.1$ is taken to be 0.068 . The effect of impurity scattering is to broaden the electron and hole levels, and to introduce additional higherorder impurity-assisted processes. The interference between the two possible channels each involving an electron- or hole-intermediate state into the indirect process turns out to subtract intensity from the low-energy side of the photons emitted and to add it to the high-energy side. Therefore the impurity-assisted process yields significant shifting and broadening of the spectral profile [11]. Taking into account the absence of the broadening caused by impurity-assisted processes as well as the absence of a noticeable shift of the PL maximum from the low-energy cut-off we conclude about the exciton-like nature of the PL which is expected for the under-CLT region and is representative for the highly homogeneous InGaAs epilayer.

Fig. 1 shows also the PL feature for InGaAs QW of smaller QW thickness (15 nm) (Sample S2). The sheet density is now $N_{s}=2.50 \cdot 10^{11} \mathrm{~cm}^{-2}$ only. One has to expect the PL feature of a FWHM value, at least, similar that for QW width equal to $20 \mathrm{~nm}$. However the PL feature observed is broader substantially, FWHM $\sim 12 \mathrm{meV}$, with clearly visible low-energy asymmetry. When the excitation intensity is elevated the obvious symmetrization of the PL profile takes place due to hot carrier contribution into the highenergy tail. The reason for smearing the low-energy cut-off at the low excitation level can be the generation of defects or excitonic contribution. Indeed the measurements of the PL response in magnetic field $(B)$ show a characteristic ripple of the PL line shape towards the higher energies, which transforms into well-resolved Landau-level structure at magnetic field as high as $B=7 \mathrm{~T}$. The Landau-level fan corresponds to the indirect off-diagonal transitions, allowed due to impurity-assisted free carrier scattering. Indeed at low temperature $(T=6 \mathrm{~K})$ only the ground level $N_{h}=0\left(N_{h}\right.$ denotes the Landau number for the hole states in magnetic field, while $N_{e}$ is the Landau number for the electron states of conduction band) is occupied. Electron-hole recombinations are expected to arise from the primary $N_{e}=0_{e} \rightarrow N_{h}=0_{h}$ transition and secondary from the off-diagonal transitions $1_{e} \rightarrow 0_{h}, 2_{e} \rightarrow 0_{h}$, etc, which are nominally forbidden by conventional selection rules $\left|N_{e}-N_{h}\right|=0$ for optical transitions in magnetic field [12]. The $6 \mathrm{~K}$ peak intensities of these forbidden transitions do not follow a Boltzmann distribution, but are determined by the off-diagonal transition matrix elements. Inasmuch the presence of these transitions give evidence for the impurity and defect appearance in the InGaAs QW. Besides we observed that the principal transition $0_{e} \rightarrow 0_{h}$ in the PL spectrum is accompanied by the low-energy band, which can be related to the exciton-like state. Really the study of the free-carrier screening effect in Shottky-gated modulation-doped $\operatorname{In}_{\mathrm{x}} \mathrm{Ga}_{1-\mathrm{x}} \mathrm{As} / \mathrm{InP} \mathrm{QW}$ has shown that exciton contribution to the PL exists up to the carrier density $N_{s} \approx 3 \cdot 10^{11} \mathrm{~cm}^{-2}$ [13]. Exciton is expected to be localized due to hole localization by alloy fluctuations. The growth of density of fluctuations in QW is revealed also by the analysis of the high-energy part of the PL feature when magnetic field is not applied. This part is accurately fitted to the theoretical line shape [11].

$$
S_{i j}(\varepsilon) \propto \int_{0}^{\varepsilon} f\left(\tau-\mu_{\mathrm{v} j}\right) f\left(\varepsilon-\tau-\mu_{\mathrm{c} i}\right) K(\varepsilon, \tau) d t,
$$

where $\varepsilon$ is the photon energy minus the effective band gap, $f$ is the Fermi distribution function, $\mu_{\mathrm{v} j}$ and $\mu_{\mathrm{c} i}$ stand for chemical potentials in the $j$-th valence and the $i$-th conduction bands, respectively. The Lorentzian $K$ accounts for the processes associated with direct transitions in $k$ space and indirect impurity-assisted processes. Following the procedure described in Ref. 11 the fit was performed for the lineshape of PL excited by a low-intensity $\mathrm{cw}$ laser. The set of parameters was derived from the fit: $E_{g}=1.394 \mathrm{eV}, \Gamma=$ $12.2 \mathrm{meV}$ ( $\Gamma$ is the FWHM value), $E_{F}=10 \mathrm{meV}$. The $E_{F}$ value obtained coincides good with that found from the selfconsistent solution of Schrodinger and Poisson equations $\left(E_{F}=10.4 \mathrm{meV}\right)$. The $E_{g}$ is somewhat shifted towards the red side (by $3 \mathrm{meV}$ ) with respect to the PL peak position. This is a consequence of 2D-carrier scattering by the charge impurities, which depends strongly on the thickness of the spacer layer. These findings give us ground to state that the high energy side of the PL profile is mainly defined by the electron-hole recombination accompanied by impurity and disorder-assisted processes, which results in broadening and blueshifting the PL line. The low-energy tail of the PL feature of sample S2 is contributed by exciton-like effects. Consequently this spectrum, in spite of lower 2DEG density with respect to that depicted for the sample S1, shows a significantly larger FWHM (by one order of magnitude), related obviously to the growth of $\mathrm{QW}$ disordering with diminishing the QW thickness. 


\section{Z.Ya. Zhuchenko et al.: Optical characterization of pseudomorphic heterostructures...}

The tendency found is held for the Sample S3 where the QW thickness is of $12 \mathrm{~nm}$ and $N_{s}=6.30 \cdot 10^{11} \mathrm{~cm}^{-2}$, being practically the same one as in Sample S1. Sample S3 shows in the low-temperature PL spectrum a pronounced knee at the Fermi energy. The FWHM value is of $16 \mathrm{meV}$. The spectrum observed is typical for strongly disordered QW. The line shape is fitted precisely to Eq. (1). A magnetoluminescence study shows that the structure of PL spectra in magnetic fields is determined both the 2DEG behavior and by the presence of localized holes. The conditions for hole localization can be explored by means of optical detection of the quantum oscillations via PL in a magnetic field $[14,15]$. The quantum oscillations in PL intensity with the Fermi sea reveal two distinct periods in $1 / B$. This behavior is attributed to the existence of two sorts of hole localization: a shallow one, primary resulting in the hole scattering, and a deep localization resulting in a $10 \mathrm{meV}$ shift of the quantum oscillations. This latter strong hole localization is caused by the alloy fluctuations, caused in part by the In content, the density of which becomes substantially enhanced in the QW of smaller widths. So, we conclude here that diminishing of the QW width favors the growth of potential fluctuations, contributed by the fluctuations in the $\operatorname{In}_{\mathrm{y}} \mathrm{Ga}_{1-\mathrm{y}} \mathrm{As}$ composition, the QW thickness, and the density of charge impurities, just in the under-CLT region of QW thicknesses.

\section{B. Raman scattering study}

The samples investigated by PL were studied then by Raman scattering technique. The unanalyzed spectra have been recorded at room temperature. The strained InGaAs QW, as thin as 10-20 nm, clad by a thicker doped AlGaAs layer is difficult to investigate here due to the $\mathrm{Al}$ content used about of $20 \%$ does not provide a sufficient distinction between GaAs-like longitudinal optical (LO) phonons in the InGaAs and barriers. While the In content is about of $10 \%$ the GaAs-like LO-phonon frequencies of the well are very close to those of bulk GaAs [16]. Fig. 2 shows such superimposed spectra for three representative samples under investigation in the spectral region of the GaAs-like LO phonon. Because the backscattering from (100) surface is considered the transversal optical (TO) modes of GaAs are nominally forbidden. TO phonon modes are allowed only in (011) and (111) backscattering. Therefore, the Raman measurements have to be made at least in two different backscattering planes to identified all the phonon modes allowed. Moreover the identification of the phonon modes in $\operatorname{In}_{\mathrm{y}} \mathrm{Ga}_{1-\mathrm{y}}$ As by Raman studies has differences as summarized by Nash et al [17]. Because the Raman spectra contain convoluted bands the lineshape analyses is needed in deconvolving the complicated spectral responses and obtaining the precise and reliable phonon frequencies [18]. The spectral feature at $v=285 \mathrm{~cm}^{-1}$ is related to the GaAs-like $\mathrm{LO}$ mode in $\mathrm{Al}_{\mathrm{x}} \mathrm{Ga}_{1-\mathrm{x}} \mathrm{As}$, which was identified following the Raman scattering studies in $n$ type and $p$-type $\mathrm{Al}_{\mathrm{x}} \mathrm{Ga}_{1-\mathrm{x}}$ As layers $[19,20]$, and it corresponds to the Al molar fraction $y=0.2$. We have also observed the peak corresponding to the AlAs-like LO phonon at $v=365 \mathrm{~cm}^{-1}$ which is broad and strongly weaker than that at $v=285 \mathrm{~cm}^{-1}$. The feature at $v=270 \mathrm{~cm}^{-1}$ is contributed by different modes: the TO phonon of GaAs due to the

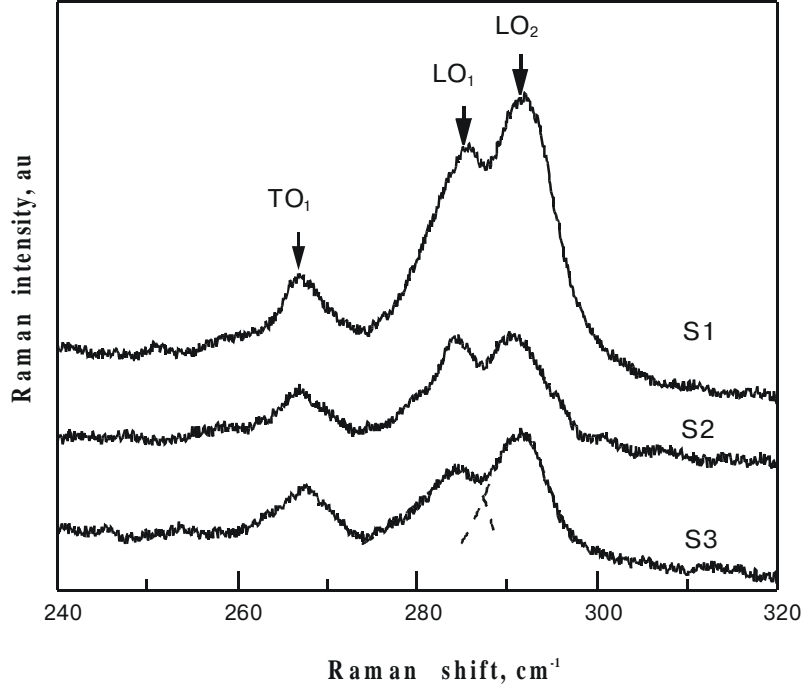

Fig. 2. Unpolarized Raman spectra of $\mathrm{Al}_{\mathrm{x}} \mathrm{Ga}_{1-\mathrm{x}} \mathrm{As} / \mathrm{In}_{\mathrm{y}} \mathrm{Ga}_{1-\mathrm{y}} \mathrm{As} / \mathrm{GaAs}$ heterostructure recorded at room temperature. The GaAs-like LO mode in InGaAs and in AlGaAs are shown by arrows. The deconvolution of superimposed peakes is shown by dashed lines. TO mode is observed at $v=$ $268 \mathrm{~cm}^{-1}$

alignment of measurement is slightly out of the backscattering geometry, observed also in the resonant Raman scattering from mixed $\mathrm{In}_{\mathrm{y}} \mathrm{Ga}_{1-\mathrm{y}}$ As crystals [21]; the coupled LO phononplasmon mode $\mathrm{L}^{-}$observed, both in $n$-type $\mathrm{Al}_{\mathrm{x}} \mathrm{Ga}_{1-\mathrm{x}} \mathrm{As}$ [19], and in the InAs epilayer with the carrier concentration $1.0 \cdot 10^{17} \mathrm{~cm}^{-3}[22]$. The deconvolution of the superimposed features in the spectral region $280-300 \mathrm{~cm}^{-1}$ and subsequent analysis of the Raman line shape was performed using the technique developed by Mintairov and Temkin [18]. In this technique the frequency dependence of the scattering efficiency for a dipole-active Stokes component of the Raman spectrum of a multimode alloy is described by

$I(\omega) \propto \operatorname{Im}\left\{-\left(1+\sum_{j} K_{j} \chi_{j}(\omega)\right)^{2} / \varepsilon(\omega, q)+\sum_{j}\left(K_{j}\right)^{2} \chi_{j}(\omega)\right\}$,

where $\varepsilon(\omega, q)$ is the dynamic dielectric function of the crystal, $j$ specifies the optical phonons, $q$ is the wave vector, $\chi_{j}(\omega)$ is the phonon susceptibilities, and the coefficients $K_{j}$ are expressed through the phonon oscillator strength and the Faust-Henry parameters [18]. The results of analysis will be presented elsewhere. Here we use the frequency of GaAs-like mode derived from the line shape analysis. It coincides closely with that found from the «spatial correlation» model [23], which using a Gaussian correlation function quantitatively explains the broadening and asymmetry of the first-order LO Raman spectrum induced by alloy potential fluctuations. Then Raman intensity can be written as [23]

$I(\omega) \propto \int_{0}^{1} \exp \left(-q^{2} L^{2} / 4\right)\left\{[\omega-\omega(q)]^{2}+\left(\Gamma_{0} / 2\right)^{2}\right\}^{-1} d^{3} q .(3)$ 


\section{Z.Ya. Zhuchenko et al.: Optical characterization of pseudomorphic heterostructures...}

Here $\Gamma_{0}$ is the FWHM of the intrinsic Raman line shape of the end-point materials, $L$ is a correlation length, and the dispersion $\omega(q)$ of the LO phonon is taken as for a onedimensional linear-chain model. Being applied to our Raman spectra Eq. (3) shows different correlation length for the GaAs-like mode in InGaAs layer, decreasing for the smaller QW widths. This reflects the decrease of ordering in the InGaAs epilayer with decrease of its thickness. The frequency shift for the allowed LO phonon caused by strain is given by [16]

$$
\omega=\left[\frac{\alpha}{\omega_{0}}+\frac{\beta}{\omega_{0}}\left(\frac{S_{11}}{S_{11}+S_{12}}\right)\right] \varepsilon,
$$

where $\varepsilon$ is the strain, $\omega_{0}$ is the zero-strain frequency of the LO mode, and $\alpha$ and $\beta$ are phonon coefficient related to frequency changes in the presence of uniform strain. $S_{11}$ and $S_{12}$ are the compliance constants, which are expressed through the elastic parameters. Fig. 3 shows the phonon frequencies found for our samples, and those for the thin strain-layered $\mathrm{In}_{\mathrm{y}} \mathrm{Ga}_{1-\mathrm{y}} \mathrm{As}$ films, grown on GaAs substrates [6]. Comparing the results we conclude that our samples should be indeed refered to the perfect epitaxy. No visible strain relaxation can be derived from the Raman scattering data: the layer with the perfect epitaxy is under the largest compressive stress, which shifts the phonon frequencies to higher energies, as can be seen from Fig. 3. The similar conclusion can be derived also from the comparison of our data with those obtained by Gilperez et al. [24] for pseudomorphic InGaAs/AlGaAs heterostructures. It should be noted here that Burns et al. [6] using the Raman technique and X-ray rocking curve technique have found the CLT for the $y \approx 0.1$ strain-layered $\operatorname{In}_{\mathrm{y}} \mathrm{Ga}_{1-\mathrm{y}} \mathrm{As}$ films grown on $\mathrm{GaAs}$ substrates has to be between $150 \mathrm{~nm}$ and $250 \mathrm{~nm}$. This latter value strongly exceeds that obtained in our analysis, about of $25 \mathrm{~nm}$, and mirrors the difference in the CLT definition. In fact the change of the layer thickness shares among numerous defects which can be generated during the growth,

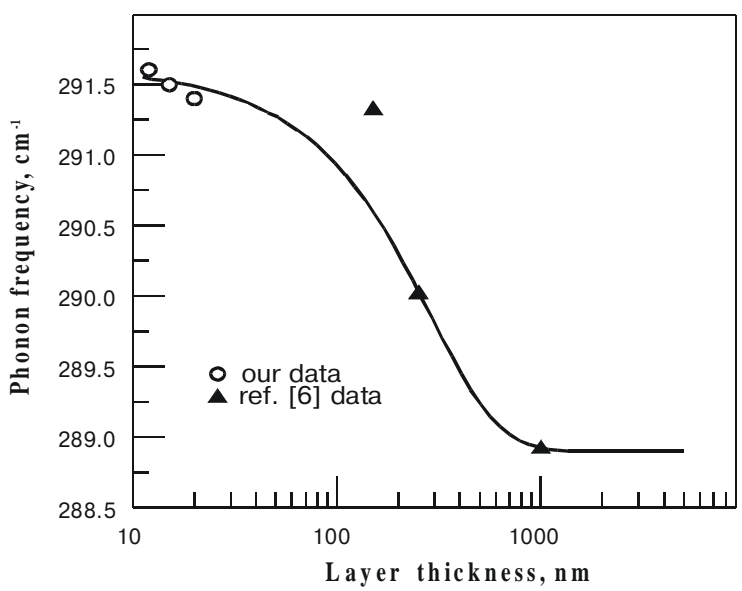

Fig. 3. The GaAs-like LO phonon frequencies in InGaAs derived from the measured Raman spectra and those from Ref. 6 versus the InGaAs layer thickness. and when the dislocations are not more threatened the epilayer quality below the CLT a new sort of defects arises which results in the layer disordering for the smaller layer thicknesses. These defects do not influence substantially the allowed phonon modes, but favor to appearance of the forbidden modes. Our Raman measurements give evidence for such conclusion.

Fig. 4 depicts the Raman spectra in the region below $200 \mathrm{~cm}^{-1}$. It is clearly seen that the spectral feature at $v=160 \mathrm{~cm}^{-1}$ gradually develops from a practically indistinguishable from the background noise level for the Sample S1 to well pronounced peak for the Sample S3. The peak around $160 \mathrm{~cm}^{-1}$ was first reported by Kakimoto and Katoda [22] when the Raman spectra from $\mathrm{Ga}_{1-\mathrm{x}} \operatorname{In}_{\mathrm{x}}$ As epitaxial layers of various compositions were studied and was considered as the disorder-activated longitudinal acoustic (DALA) phonon of $\mathrm{Ga}_{1-\mathrm{x}} \mathrm{In}_{\mathrm{x}} \mathrm{As}$, because its intensity was at maximum near the middle-range composition. Then the DALA phonon was identified in the resonant Raman scattering at the saddle-point singularity in $\mathrm{In}_{\mathrm{x}} \mathrm{Ga}_{1-\mathrm{x}} \mathrm{As}$ which was of a very weak intensity at the $2.41 \mathrm{eV}$ laser energy, but become enhanced near the $E_{1}$ gap for the $\operatorname{In}_{0.24} \mathrm{Ga}_{0.76} \mathrm{As}$ sample [21]. In the case of $\operatorname{In}_{0.53} \mathrm{Ga}_{0.47} \mathrm{As}$ this feature was observed as a strong one far from resonance and become further enhanced near the $E_{1}$ gap. We find here that DALA phonon develops clearly in the sample, where the PL response also signalizes about $\mathrm{QW}$ disordering. So the correlation between the PL and Raman spectra observed in our $\mathrm{Al}_{\mathrm{x}} \mathrm{Ga}_{1-\mathrm{x}} \mathrm{As} / \mathrm{In}_{\mathrm{y}} \mathrm{Ga}_{1-\mathrm{y}} \mathrm{As} / \mathrm{GaAs}$ heterostructures appears to be very indicative for the QW quality in the region of QW widths far beyond the CLT. Other peaks observed below $v=160 \mathrm{~cm}^{-1}$ can be assigned in part to the disorderactivated compound modes, and in part their origin is not yet explored. The peak at $v=160 \mathrm{~cm}^{-1}$ can be contributed also by the 2TA (transverse acoustic) mode of InGaAs, activated by disorder.

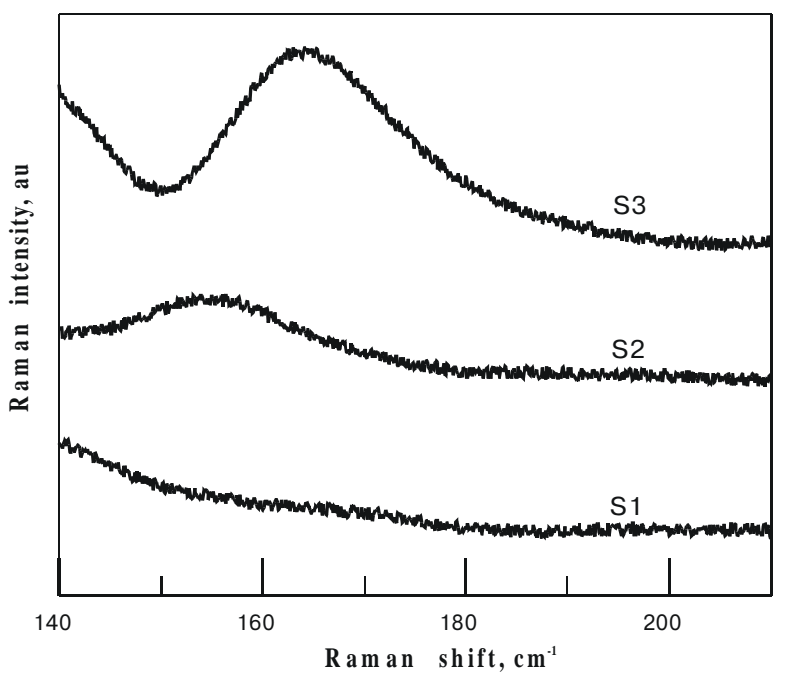

Fig. 4. Raman spectra for three representative samples in the spectral region of defect-activated acoustic modes of InGaAs. 


\section{Z.Ya. Zhuchenko et al.: Optical characterization of pseudomorphic heterostructures...}

\section{Conclusions}

Summarizing the results of PL and Raman scattering studies of modulation-doped pseudomorphic strained-layer $\mathrm{Al}_{\mathrm{x}} \mathrm{Ga}_{1-\mathrm{x}} \mathrm{As} / \mathrm{In}_{\mathrm{y}} \mathrm{Ga}_{1-\mathrm{y}} \mathrm{As} / \mathrm{GaAs}$ heterostructures we have established the correlation between the PL line shape changes and the Raman spectra modification when the QW width is below the critical thickness estimated to be $25 \mathrm{~nm}$ for $y=0.1$. The PL feature observed for the InGaAs QW width equal to $20 \mathrm{~nm}$ as the extremely narrow exciton-like peak with the FWHM equal $1.5 \mathrm{meV}$ at low temperature $(T=6 \mathrm{~K})$ transforms into a broad band of the FWHM equal $16 \mathrm{meV}$ when the QW width reaches the value about of $12 \mathrm{~nm}$. This effect is connected with worsening the quality of InGaAs epilayer due to growth of fluctuation density; contributed by the fluctuations in the $\mathrm{In}_{\mathrm{y}} \mathrm{Ga}_{1-\mathrm{y}}$ As composition, the QW thickness, and the density of charge impurities immediately in the under-CLT region of QW thicknesses. The PL line broadening is accompanied by the modifications in Raman spectra. A new line arising at the spectral position $v=160 \mathrm{~cm}^{-1}$ is related to the longitudinal acoustic mode of $\operatorname{In}_{\mathrm{y}} \mathrm{Ga}_{1-\mathrm{y}} \mathrm{As}$ induced by increased disorder of the narrower QW. This line seems to be more indicative for the evaluation of disordering degree than the conventional GaAs-like mode of InGaAs layer in the region of the layer thicknesses far below the CLT. Inasmuch the high quality homogeneous epilayer is expected to get only for a certain range of thicknesses placed below the CLT. The range depends likely on the $\mathrm{In}_{\mathrm{y}} \mathrm{Ga}_{1-\mathrm{y}}$ As composition and will be the subject of further investigation.

\section{Acknowledgments}

This work is supported by NATO linkage grant. Authors are indebted to V.O.Yukhimchuk for his assistance during Raman scattering measurements.

\section{References}

1. S. Adachi, Material parameters of $\operatorname{In}_{1-\mathrm{x}} \mathrm{Ga}_{\mathrm{x}} \mathrm{As}_{\mathrm{y}} \mathrm{P}_{1-\mathrm{y}}$ and related binaries // J. Appl. Phys. 53, pp. 8775-8792 (1982).

2. M. J. Ekensterdt, T. G. Andersson, and S. M. Wang, Temperaturedependent relaxation and growth phenomena in strained $\operatorname{In}_{\mathrm{x}} \mathrm{Ga}_{1-\mathrm{x}} \mathrm{As}$ layers grown on GaAs // Phys. Rev. B 48, pp. 5289-5299 (1993).

3. L. R. Tessler, C. Hermann, G. Lampel, Y. Lassailly, C. Fontaine, E. Daran, and A. Munoz-Yague, Highly polarized photoluminescence from 2-mm-thick strained GaAs grown on $\mathrm{CaF}_{2} / /$ Appl. Phys. Lett. 64, pp. 895-897 (1994).

4. J. W. Matthews and A. E. Blakeslee,Defects in epitaxial multilayers I. Misfit dislocations // J. Cryst. Growth 27, pp. 118-125 (1974).

5. M. Gal, P. J. Orders, B. F. Usher, M. J. Joyce, and J. Tann, Observation of compressive and tensile strains in InGaAs/GaAs by photoluminescence spectroscopy // Appl. Phys. Lett. 53, pp. 113-115 (1988)

6. G. Burns, C. R. Wie, F. H. Dacol, G. D. Pettit, and J. M. Woodall, Phonons shifts and strains in strain-layered $\left(\mathrm{Ga}_{1-\mathrm{x}} \mathrm{In}_{\mathrm{x}}\right)$ As // Appl. Phys. Lett. 51, pp. 1919-1921 (1987).
7. P. S. Pizani, T. M. Boschi, F. Lanciotti Jr., J. Groenen, R. Carles, P. Maigne, and M. Gendry, Alloying effects on the crytical layer thickness in $\mathrm{In}_{\mathrm{x}} \mathrm{Ga}_{1-\mathrm{x}} \mathrm{As} / \mathrm{InP}$ heterostructures analyzed by Raman scattering // Appl. Phys. Lett. 72, pp. 436-438 (1998).

8. O. Brafman, D. Fekete, and R. Sarfaty, Phonon study of strained InGaAs layers // Appl. Phys. Lett. 58, pp. 400-402 (1991).

9. S. Wang, T. Andersson, V. Kulakovskii, and J. Yao, Critical layer thickness in $\operatorname{In}_{\mathrm{x}} \mathrm{Ga}_{1-\mathrm{x}} \mathrm{As} / \mathrm{GaAs}$ quantum wells studied by photoluminescence and transmission electron microscopy // Superlattices and Microstructures 9, pp. 123-126 (1991).

10. H. Kissel, U. Mueller, C. Walther, W. T. Masselink, Yu. I. Mazur, G. G. Tarasov, and Z. Ya. Zhuchenko,Peculiarities of photoluminescence in pseudomorphic modulation-doped $\mathrm{Al}_{0.2} \mathrm{Ga}_{0.8} \mathrm{As}_{2} / \mathrm{In}_{0.1} \mathrm{Ga}_{0.9} \mathrm{As} / \mathrm{GaAs}$ quantum wells // Phys. Rev. B 58, pp. $4754-4760$ (1998).

11. S. K. Lyo and E. D. Jones, Photoluminescence line shape in degenerate semiconductor quantum wells // Phys. Rev. B 38, pp. 4113-4119 (1988).

12. S. K. Lyo, E. D. Jones, and J. F. Klem, Breaking of the usual selection rule for magnetoluminescence in doped semiconductor quantum wells // Phys. Rev. Lett. 61, pp. 2265-2268 (1988).

13. M. S. Skolnick, K. J. Nash, P. R. Tapster, W. Mowbray, S. J. Bass and A. D. Pitt, Free-carrier screening of the interaction between excitons and longitudinal-optical phonons in $\operatorname{In}_{\mathrm{x}} \mathrm{Ga}_{1-\mathrm{x}}$ As-InP quantum wells / / Phys. Rev. B 35, pp. 5925-5928 (1987).

14. I. A. Buyanova, W. M. Chen, A. V. Buyanov, W. G. Bi, and C. W. Tu, Optical detection of quantum oscillations in InP/InGaAs quantum structures // Appl. Phys. Lett. 69, pp. 809-811 (1996).

15. G. G. Tarasov, U. Mueller, Yu. I. Mazur, H. Kissel, Z. Ya. Zhuchenko, C.Walther, and W. T. Masselink, Direct investigation of localized hole states in pseudomorphic modulation-doped $\mathrm{Al}_{\mathrm{x}} \mathrm{Ga}_{1-\mathrm{x}} \mathrm{As} / \mathrm{In}_{\mathrm{y}} \mathrm{Ga}_{1-\mathrm{y}} \mathrm{As} /$ GaAs heterostructures by optical detection of quantum oscillations // Phys. Rev. B 58, pp. $4733-4739$ (1998).

16. F. Iikawa, F. Cerdeira, C. Vazquez-Lopez, P. Motisuke, A. Sacilotti, A. P. Roth, and R. A. Mazut, Raman scattering from InGaAs/GaAs strained-layer superlattices // Solid State Commun. 68, pp. 211-214 (1988).

17. K. J. Nash, M. S. Skolnick, and S. J. Bass, Electron-phonon interactions in indium gallium arsenede // Semicond. Sci.Technol. 2, pp. 329-336 (1987).

18. A. M. Mintairov and H. Temkin, Lattice vibrations and phonon-plasmon coupling in Raman spectra of $p$-type $\mathrm{In}_{0.53} \mathrm{Ga}_{0.47} \mathrm{As} / /$ Phys. Rev. $B$ 55, pp. 5117-5123 (1997).

19. T. Yuasa, S. Naritsuka, M. Mannoh, K. Shinozaki, K. Yamanaka, Y. Nomura, M. Mihara, and M. Ishii, Raman scattering from coupled plasmon-LO-phonon modes in $n$-type $\mathrm{Al}_{\mathrm{x}} \mathrm{Ga}_{1-\mathrm{x}} \mathrm{As} / /$ Phys. Rev. B 33, pp. 1222-1232 (1986).

20. T. Yuasa and M. Ishii, Raman scattering study of coupled hole-plasmon-LO-phonon modes in p-type GaAs and p-type $\mathrm{Al}_{\mathrm{x}} \mathrm{Ga}_{1-\mathrm{x}} \mathrm{As} / /$ Phys. Rev. B 35, pp. $3962-3970$ (1987).

21. K. P. Jain, R. K. Soni, S. C. Abbi, and M. Balkanski, Resonant Raman scattering at the saddle-point singularity in $\operatorname{In}_{\mathrm{x}} \mathrm{Ga}_{1-\mathrm{x}} \mathrm{As} / /$ Phys. Rev. B 32, pp. 1005-1008 (1985).

22. K. Kakimoto and T. Katoda, Raman spectra from $\mathrm{Ga}_{1-\mathrm{x}} \mathrm{In}_{\mathrm{x}}$ As epitaxial layers on GaAs and InP substates // Appl. Phys. Lett. 40, pp. 826-828 (1982).

23. P. Parayanthal and F. H. Pollak, Raman scattering in alloy semiconductors: «Spatial correlation» model // Phys. Rev. Lett. 52, pp. 18221825 (1984).

24. J. M. Gilperez, F. Gonzalez-Sanz, E. Calleja, E. Munoz, J. M. Calleja, N. Mestres, J. Castagne, and E. Barbier, Photoluminescence and Raman analysis of strain and composition in InGaAs/AlGaAs pseudomorphic heterostructures // Semicond. Sci. Technol. 7, pp. 562566 (1992). 